\title{
Office Workers' Perceived Barriers and Facilitators to Taking Regular Micro-Breaks at Work: a Diary-Probed Interview Study
}

\author{
Yitong Huang ${ }^{1}$, Steve Benford ${ }^{1}$, Hilde Hendrickx ${ }^{2}$, Rob Treloar ${ }^{2}$, and Holly Blake \\ ${ }^{1}$ The University of Nottingham, Nottingham, United Kingdom \\ \{yitong.huang, steve.benford, holly.blake\}@nottingham.ac.uk \\ 2Unilever R\&D, Sharnbrook, United Kingdom \\ \{hilde.hendrickx, rob.treloar\}@unilever.com
}

\begin{abstract}
Research has suggested regular breaks in sedentary office work are important for health, wellbeing and long-term productivity. Although many computerized break reminders exist, few are based on user needs and requirements as determined by formative research. This paper reports empirical findings from a diaryprobed interview study with 20 office workers on their perceived barriers and facilitators to taking regular micro-breaks at work. This work makes two contributions to the Persuasive Technology (PT) community: a diagnosis of the full range of determinants and levers for changing office work break behaviours; a demonstration of applying the Behaviour Change Wheel (BCW), an intervention development framework originating from Health Psychology, to elicit theory-based design recommendations for a potential PT.
\end{abstract}

Keywords. Workplace sedentary behaviour · Requirement elicitation method

\section{Introduction}

It is well recognized in health sciences that too much sitting, especially prolonged sitting ( $>60 \mathrm{~min}$ ) without breaks, is associated with increased risks for metabolic syndrome, obesity, cardiovascular diseases and a range of other conditions [19], regardless of the amount of exercise [15]. Occupational sitting is a health hazard for sedentary office workers [17], who would benefit from hourly micro-breaks (3-5 minutes), that involve light physical activities even as simple as walking to the kitchen and refilling a mug, to alleviate the metabolic dysfunction caused by long periods of sitting [14]. In addition to physical health benefits, management literature has suggested micro-breaks are essential in maintaining employees' psychological wellbeing and energy level [9].

There exist many computerized break reminders that come in a variety of forms, ranging from popup windows on workstation screens and browser plug-ins based on the Pomodoro Technique [4], to wearable gadgets with vibrating inactivity alerts (e.g. Jawbone, Apple Watch). Previous Human-Computer Interaction (HCI) work has approached the

This is the version prepared by the author. The final publication is available at Springer via http://dx.doi.org/10.1007/978-3-319-55134-0 12 
problem from a cognitive perspective, in relation to interruption and attention management in organizational contexts $[11,12]$; some has led to the identification of opportune moments for delivering persuasive messages during working hours [21].

However, to date, there is a lack of research looking into the problem through the lens of behaviour change. Just as a doctor needs to diagnose a patient's problem before writing out a prescription, PT designers will also benefit from carrying out a thorough behavioural diagnosis, which should reveal all behavioural facets that require modifications. In view of that, we conducted a study to answer the question of what facilitates and hinders microbreaks at work from office workers' perspectives. The following section will introduce the methodological framework that guides our study. Afterwards, we present the study method, findings and a recommended selection of intervention functions and behaviour change techniques. The paper concludes with a discussion of limitations and practical recommendations.

\section{Framework Guiding the Study: Behaviour Change Wheel}

There are numerous theories that predict and explain behaviour change, presenting PT designers with the challenge of selecting theories most appropriate and relevant to the problem under investigation. It is positive that an increasing number of PT studies are underpinned by psychology theories, such as goal-setting theory $[5,10]$ and Transtheoretical Models [5]. However, the selection of theory is still heavily reliant on the designer's instinctive understanding of the behaviour and existing knowledge of psychology theories, rather than a systematic and theoretically guided process [13]. Such an approach excludes potentially relevant and viable theories and persuasive strategies [7]. For instance, the widely known Transtheoretical Model and Health Belief Model are increasingly questioned for their failure to address automatic motivational factors (e.g. impulses, habits, and emotions) that can be powerful drives for some behaviours [20].

To guide the process of selecting and translating theories into intervention design, several intervention development frameworks have been proposed, although most of these have been judged as not sufficiently coherent, comprehensive or well-linked to a model of behaviour, according to Michie et al. [13], who consequently developed the Behaviour Change Wheel (BCW).

The BCW is underpinned by a behavioural model at the centre called "COM-B", which breaks down behavioural problems in terms of possible deficits in three aspects (with two subcomponents in each aspect), namely Capability (psychological and physical), Opportunity (physical and social) and Motivation (automatic and reflective). The BCW also summarises nine intervention functions (e.g. education, coercion, restriction, environmental restructuring) that address one or more of the six COM-B components. As those intervention functions are defined in very general terms, they are further delineated with 93 Behaviour Change Techniques (BCTs) (e.g. "habit formation", "social reward"), which are irreducible active ingredients within an intervention package.

In the PT community, the Fogg Behaviour Model (FBM) [6] is probably the most wellknown model for analysing behaviours. We believe COM-B has at least two advantages over FBM as a behavioural model. First, FBM is merely a model for analysing behaviours, 
whereas the COM-B is situated within the $\mathrm{BCW}$, which would translate the behavioural analysis into the design of specific intervention features that target those COM-B components to produce the change. Second, developers of the $\mathrm{BCW}$ have made deliberate efforts to link each COM-B component with one or more domains under the Theoretical Domain Framework (TDF), an integrative framework that groups behaviour change theories into 14 domains based on overlapping constructs [3]. In a nutshell, the TDF can be seen as a variant of the COM-B model with a more fine-grained classification of facets underlying behaviours. The compatibility with TDF is valuable for two reasons. First, the TDF is a validated model already used by psychologists to elicit and analyse data in behavioural diagnosis [7, 8]. Second, while Fogg also attempts to expand on the FBM with several subcomponents (e.g. pleasure/pain, social deviance), the TDF covers a much wider range of psychological mechanisms in a more systematic manner (i.e. based on overlaps in theoretical constructs). In the next section, we will explain how the COM-B and TDF have been used for the elicitation and analysis of data in our study.

\section{Method}

\subsection{Participants and Recruitment}

The study was promoted via posters and news bulletins at the University of Nottingham, and staff mailing lists for two non-profit organisations (NPO). Office workers spending at least 2 days of the week in sedentary (chair-bound most of the time) or semi-sedentary (intermittently chair-bound and moving around but without substantial walking or physical labour) jobs volunteered to participate and were directed to an online screening questionnaire; we excluded office workers who felt that they had no discretion over timing of micro-breaks, because changing those peoples' patterns most likely required organisational/policy change and were thus beyond the scope of this work. As a result, we recruited 20 eligible participants $(\mathrm{F}=12, \mathrm{M}=8$, mean age $=35.4 \pm 11.4 \mathrm{yrs}$. old $)$, who were employed in a variety of office-based roles including project management, communication, IT support, clinical research admin, filmmaking, teaching and research.

\subsection{Procedure and Materials}

Data collection consisted of two main stages, a 2-day diary period and a 1-hour interview session. Each participant attended a 15-min briefing session with the researcher, at which they consented to participate in the study, answered demographic questions and were given a diary pad together with verbal and written instructions of the diary protocol. Participants were requested to record any two workdays in the following week as continuous series of sitting and break episodes, and note down the time whenever they left and returned to seat (Fig.1. left). Participants were told the definition of breaks as any "interruption in sitting". For each break, participants needed to take a photo of the physical context of this break and complete a "work break experience" form, which elicited in-situ responses about the decision and experience relating to the break; for instance, the form asked participants to 
complete sentences such as the following, "I wish I had taken this break earlier/later (delete where inappropriate), because...".

When participants expected an unusual day (e.g. fieldwork, conferences or travels that were not part of everyday routines), they were asked to keep the diary the following workday. The diary was collected once 2 full workdays were recorded. The recorded lengths of workdays in the study ranged from 6 hours $48 \mathrm{~min}$ to 10 hours $51 \mathrm{~min}$. Semi-structured interviews based on the TDF (see Table 1) were conducted within a week following the collection of diaries. Prior to each interview, the researcher reviewed all diaries and marked specific events or behavioural patterns pertinent to theoretical constructs [3] for elaboration and clarification in interviews. The researcher also transcribed diary entries onto a spreadsheet, which was then used to produce a visual representation of the sit-break pattern for each participant (Fig. 1.). Moreover, the researcher also produced two graphs with dummy data illustrating two disparate workstyles, namely "Workstyle 1" that has breaks every 2- 3 hours, and "Workstyle 2" that features a micro-break in every $45 \mathrm{~min}$ to $60 \mathrm{~min}$ of sitting. Those dummy examples were meant to facilitate discussion on the pros and cons of different workstyles. Starting from Participant 8, a ranking of participants based on their accumulative durations of prolonged sitting episodes per day was produced and brought to interviews.
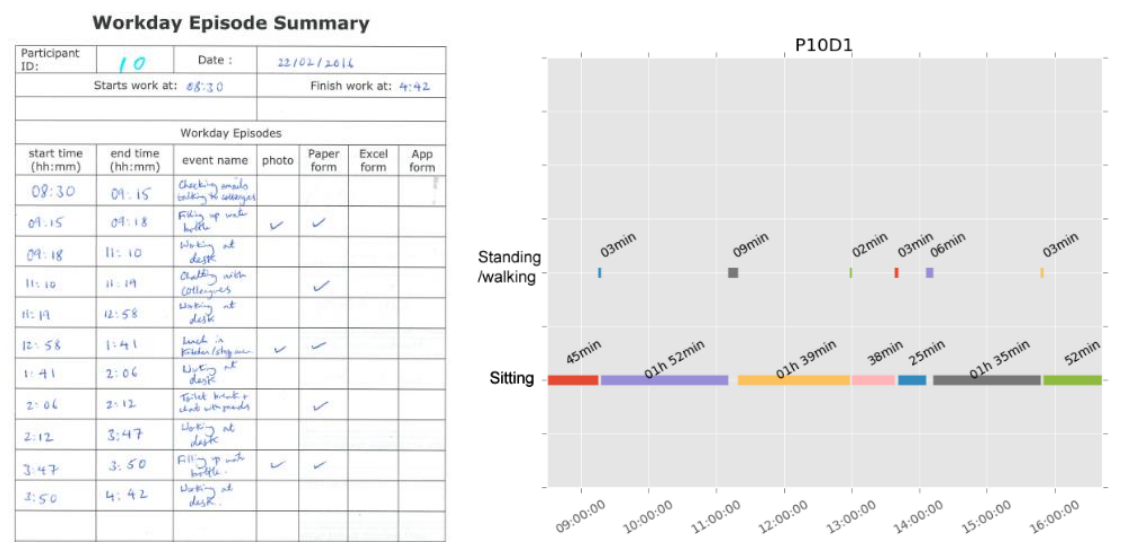

Fig. 1. A sit-break pattern graph produced based on diary entries

\subsection{Data Analysis}

To retain links between quotes and individual respondents, the Framework Approach to qualitative analysis [18] was used - the first author read through all interview transcripts and coded relevant quotes onto TDF domains and COM-B components. Coding was then reviewed by two health psychologists familiar with the $\mathrm{BCW}$, after which minor revisions were made. Afterwards, the first author summarised sub-themes on perceived barriers and facilitators emerging under each domain and counted their frequency. The BCW guide [13] that links COM-B/TDF domains to intervention functions was used to select intervention functions and BCTs likely to be effective. 
Table 1. Interview topic guide based on COM-B and TDF

\begin{tabular}{|c|c|c|}
\hline $\begin{array}{l}\mathrm{CO} \\
\mathrm{M}-\mathrm{B} \\
\end{array}$ & $\begin{array}{l}\text { TDF } \\
\text { Domain }\end{array}$ & Eliciting Questions \\
\hline \multirow{6}{*}{ 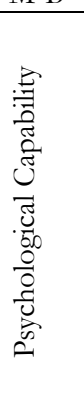 } & $\begin{array}{l}\text { Know- } \\
\text {-ledge }\end{array}$ & $\begin{array}{l}\text { What do you think would be the ideal work break pattern? } \\
\text { How did you get to know about it? What do you think it is based on? }\end{array}$ \\
\hline & Skills & How easy or difficult would you find it to follow workstyle 2 ? \\
\hline & \multirow{2}{*}{$\begin{array}{l}\text { Memory, at- } \\
\text { tention and } \\
\text { decision pro- } \\
\text { cess }\end{array}$} & $\begin{array}{l}\text { If it were not for this study, would you always have an idea of how long } \\
\text { you've been sitting for? }\end{array}$ \\
\hline & & $\begin{array}{l}\text { Was that break a conscious decision? What were your thoughts when } \\
\text { you decided that? }\end{array}$ \\
\hline & \multirow[t]{2}{*}{$\begin{array}{l}\text { Behavioural } \\
\text { regulation }\end{array}$} & $\begin{array}{l}\text { Do you set any rules for yourself regarding when you should stand up } \\
\text { and move around? }\end{array}$ \\
\hline & & $\begin{array}{l}\text { Do you have a system to help monitor whether you have taken regular } \\
\text { breaks on workdays? }\end{array}$ \\
\hline \multirow{7}{*}{ 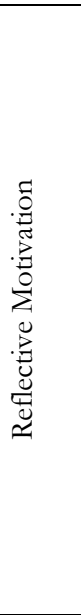 } & $\begin{array}{l}\text { Belief about } \\
\text { conse- } \\
\text { quences }\end{array}$ & $\begin{array}{l}\text { (Show two work break patterns) Which of the two do you think is bet- } \\
\text { ter? Why? How convinced are you? (prompts: in terms of health, } \\
\text { productivity, social and mood consequences respectively) }\end{array}$ \\
\hline & $\begin{array}{l}\text { Belief about } \\
\text { capabilities }\end{array}$ & $\begin{array}{l}\text { Would you find it helpful to have a piece of technology that 1) moni- } \\
\text { tors and displays your sitting time 2) triggers inactivity alerts 3) gives } \\
\text { you feedback on your break pattern at the end of each day? }\end{array}$ \\
\hline & Optimism & $\begin{array}{l}\text { How confident do you feel about breaking up your sitting with regular } \\
\text { micro-breaks? }\end{array}$ \\
\hline & \multirow[t]{2}{*}{ Goal } & Do you want to change your current sitting patter in any way? \\
\hline & & $\begin{array}{l}\text { Compared to the goal of completing your work, to what extent is hav- } \\
\text { ing a healthier work pattern a priority for you? What about in the long- } \\
\text { term? }\end{array}$ \\
\hline & Intention & Is taking regular micro-breaks something you intend to do? \\
\hline & $\begin{array}{l}\text { Social/pro- } \\
\text { fessional role } \\
\text { and identity }\end{array}$ & $\begin{array}{l}\text { Is sitting and working at desk for a prolonged period of time consistent } \\
\text { with your professional standard? } \\
\text { To what extent do you see yourself as someone conscious of the health } \\
\text { impacts of your own lifestyle choices? }\end{array}$ \\
\hline \multirow{3}{*}{ 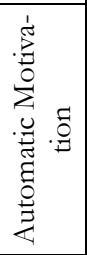 } & \multirow[t]{2}{*}{$\begin{array}{l}\text { Reinforce- } \\
\text { ment }\end{array}$} & $\begin{array}{l}\text { Would you say that generally you are in the habit of sitting for over } 60 \\
\text { minutes/taking regular breaks? If not, what would be helpful in devel- } \\
\text { oping/breaking that routine/habit? }\end{array}$ \\
\hline & & $\begin{array}{l}\text { Do you feel your break time experience is rewarding enough at the mo- } \\
\text { ment? }\end{array}$ \\
\hline & Emotion & $\begin{array}{l}\text { Does taking a break evoke an emotional response? Is the decision to } \\
\text { take breaks influenced by any emotion? }\end{array}$ \\
\hline \multirow{3}{*}{ 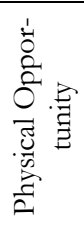 } & \multirow{3}{*}{$\begin{array}{l}\text { Environ- } \\
\text { mental con- } \\
\text { text and re- } \\
\text { sources }\end{array}$} & What break facilities would you like to have access to? \\
\hline & & $\begin{array}{l}\text { Are there any other factor that facilitates or hinders micro-breaks? (e.g. } \\
\text { nature and structure of work that demands long period of concentra- } \\
\text { tion to get into the flow/for consistent outcome) }\end{array}$ \\
\hline & & $\begin{array}{l}\text { How do you like the idea of having a smart cup in the office that } \\
\text { prompts breaks? }\end{array}$ \\
\hline \multirow{4}{*}{ 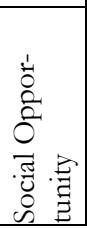 } & \multirow{4}{*}{$\begin{array}{l}\text { Social influ- } \\
\text { ences }\end{array}$} & What's the culture of taking breaks in your workplace? \\
\hline & & How do your manager/supervisor perceive taking regular breaks? \\
\hline & & $\begin{array}{l}\text { Would you feel part of a "crowd" or any social pressure if you follow } \\
\text { workstyle 2? }\end{array}$ \\
\hline & & $\begin{array}{l}\text { How do you find the ranking I showed you? Would you be motivated } \\
\text { by that? }\end{array}$ \\
\hline
\end{tabular}




\section{$4 \quad$ Results}

Table 2. Summary of sub-themes mapped onto COM-B and TDF with frequency counts

\begin{tabular}{|c|c|c|c|}
\hline $\begin{array}{l}\mathrm{CO} \\
\mathrm{M}-\mathrm{B}\end{array}$ & $\begin{array}{l}\text { TDF do- } \\
\text { mains }\end{array}$ & Sub-themes about perceived facilitators and barriers & $\begin{array}{l}\text { FQ. } \\
(\mathrm{n}= \\
20)\end{array}$ \\
\hline \multirow{6}{*}{ 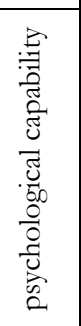 } & $\begin{array}{l}1 . \\
\text { Knowledge }\end{array}$ & $\begin{array}{l}\text { unsure about the optimum break interval or scientific rationale } \\
\text { behind it }\end{array}$ & 16 \\
\hline & \multirow{3}{*}{$\begin{array}{l}\text { 2. Memory, } \\
\text { attention } \\
\text { and decision }\end{array}$} & forget to take breaks & 18 \\
\hline & & forget having taken breaks and for how many times & 20 \\
\hline & & complex decision process involving multiple factors & 15 \\
\hline & \multirow{2}{*}{$\begin{array}{l}\text { 3. Behav- } \\
\text { ioural regula- } \\
\text { tion }\end{array}$} & need to break existing habit and apply new "if-then" rules & 13 \\
\hline & & $\begin{array}{l}\text { need a system to ease self-monitoring of break behaviours, pro- } \\
\text { vide feedback on my behaviours and progress over time }\end{array}$ & 12 \\
\hline \multirow{9}{*}{ 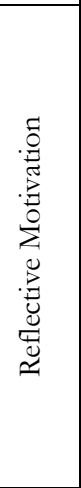 } & \multirow{4}{*}{$\begin{array}{l}\text { 4. Belief } \\
\text { about conse- } \\
\text { quences }\end{array}$} & $\begin{array}{l}\text { health consequences: } \\
\text { a. believe micro-breaks have independent health benefits }\end{array}$ & $\underline{11}$ \\
\hline & & b. unconvinced of benefits of micro-breaks & $\underline{9}$ \\
\hline & & $\begin{array}{l}\text { productivity: } \\
\text { a. overall speaking, workstyle } 2 \text { is more productive than } 1\end{array}$ & 13 \\
\hline & & b. workstyle 2 is particularly unproductive for some tasks & 14 \\
\hline & $\begin{array}{l}\text { 5. Belief } \\
\text { about capa- } \\
\text { bilities }\end{array}$ & $\begin{array}{l}\text { a technology with automatic tracking function, prompts/cues } \\
\text { and visual feedback would give me confidence in improving my } \\
\text { break pattern, despite some difficulty at the beginning }\end{array}$ & 12 \\
\hline & 6. Goals & Workstyle 2 is a goal with changing priority and accessibility & 11 \\
\hline & \multirow{3}{*}{ 7. Intentions } & a. having taken an action towards the target behaviour change & $\underline{7}$ \\
\hline & & b. contemplating or preparing for the target behaviour change & $\underline{10}$ \\
\hline & & c. no intention to change even after participating in the study & $\underline{3}$ \\
\hline \multirow{4}{*}{ 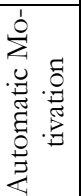 } & \multirow{2}{*}{$\begin{array}{l}\text { 8. Reinforce- } \\
\text { ment }\end{array}$} & a. existing habits that contribute to regular break behaviours & 12 \\
\hline & & b. existing habits that contribute to prolonged sitting & 12 \\
\hline & \multirow[b]{2}{*}{ 9. Emotion } & breaks evoke positive affect or remove negative affect & 12 \\
\hline & & $\begin{array}{l}\text { breaks evoke or do not help with negative affect, or negative af- } \\
\text { fect hinders micro-break behaviours }\end{array}$ & 10 \\
\hline \multirow{5}{*}{ 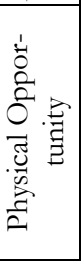 } & \multirow{5}{*}{$\begin{array}{l}\text { 10. Environ- } \\
\text { mental con- } \\
\text { text and re- } \\
\text { sources }\end{array}$} & $\begin{array}{l}\text { the organisational culture and climate } \\
\text { a. encourages micro-breaks and active work culture }\end{array}$ & $\underline{10}$ \\
\hline & & b. neither encourages or discourages breaks despite flexibility & $\underline{7}$ \\
\hline & & c. discourages regular breaks and I feel I am being watched & $\underline{3}$ \\
\hline & & $\begin{array}{l}\text { heavy workload and tight deadlines impel me to sit and work } \\
\text { continuously longer than I would like to }\end{array}$ & 15 \\
\hline & & need prompts/cues; existing reminders have flaws in design & 20 \\
\hline \multirow{4}{*}{ 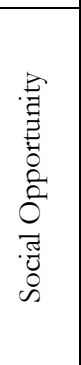 } & \multirow{4}{*}{$\begin{array}{l}\text { 11. Social in- } \\
\text { fluences }\end{array}$} & $\begin{array}{l}\text { direct social interactions that } \\
\text { a. prompt breaks (e.g. social support, invite each other for } \\
\text { breaks, short or walking meetings) }\end{array}$ & $\underline{11}$ \\
\hline & & $\begin{array}{l}\text { b. inhibit breaks (e.g. bring drinks back to seat for each other, } \\
\text { prolonged meetings without comfort breaks) }\end{array}$ & $\underline{5}$ \\
\hline & & $\begin{array}{l}\text { social norm and social pressure: } \\
\text { a. other people are good at taking regular micro-breaks and } \\
\text { there is no pressure on sitting down to work }\end{array}$ & $\underline{10}$ \\
\hline & & $\begin{array}{l}\text { b. other people sit quite a lot and prolonged sitting is perceived } \\
\text { as hard-working }\end{array}$ & 7 \\
\hline
\end{tabular}


The analysis revealed facilitators and barriers to the target behaviour (i.e. break prolonged sitting with hourly micro-breaks) in 11 associated TDF domains and 5 relevant COM-B components, as summarised in Table 2 . The last column presents frequency with which each belief was mentioned among 20 interviewees. A domain was judged to be relevant, if it had prevalent belief(s) stated by over 10 participants (bold), or if several competing beliefs were raised by over 10 participants (underlined).

\subsection{Factors underlying micro-break behaviours:}

In this subsection, we highlight four important high-level themes, summarised from subthemes in Table 2. For each quote, we report the respondent's gender, job and employer/work setting(s) in bracket.

Variability of reflective motivation to take breaks among participants . First, we found a high variability across 20 participants in their beliefs about consequences of different work break styles. Only 11 participants strongly believed in the health benefits of regular micro-breaks, whereas the rest believed that prolonged sitting only had a limited impact on themselves, because they were still young, or/and that they had sufficient physical activities outside of work (e.g. 10,000 step/day) to outbalance the adverse impact of occupational sitting. When it came to productivity, 13 participants believed micro-breaks had an overall beneficial effect on work, which was a potential motivator for micro-break behaviours; however, there was also the concern that micro-breaks were particularly unproductive for certain tasks (e.g. programming, writing), as mentioned by 14 participants. In relation to beliefs about consequences, limited or uncertain knowledge about the optimum break interval or scientific rationale behind the recommendation was another barrier to the target behaviour, e.g. "only because my watch tells me every hour to get up...but until you send a non-Apple-paid doctor in front of me and tell me you absolutely should stand up every hour. Then I would do it" (P16, male, filmmaker, NPO).

This was mirrored by the variability in participants' intention to adopt a more regular break pattern. 9 out of 20 participants had clearly made the decision and some efforts to improve their break patterns before participating in the study (e.g. P2 (male, project coordinator, NPO): "I looked at this about two months ago, just apps for the MacBook for reminding you to take breaks.”); 10 participants had started contemplating or preparing for the target behaviour change since participating in the study (e.g. P12 (female, clinical research admin, university/healthcare): "I think it wasn't long enough to change my behaviour then. But now I'm perhaps more aware of making sure I get up and make a drink... But even though I know I would be healthier...it's like I need that motivation to actually do it."); 2 participants had expressed no intention to change by the end of the debriefing interview. Admittedly, our study had a self-selected sample of participants who were potentially better-educated and more health-aware than average office workers. However, this variability still suggested that theoretical constructs in the intentions domain, such as stages of change [16], was still relevant to and predictive of the target behaviour to a certain extent. 
Notably, participants' motivations to break up their sitting with micro-breaks fluctuated at different times, suggesting the relevance of the goals domain, and its distinction from the intentions domain. According to the TDF, an intention refers to a conscious decision or resolve to act in a certain way, which is relatively stable, whereas a goal is the mental representation of a desired end state, which can become more or less accessible to the person depending on the context [3]. In our sample, 11 participants perceived the hourly break pattern as a desirable end state (i.e. a goal), but the goal priority can change depending on the work state, e.g. "I had several documents open, trying to match things up, I didn't want to interrupt my train of thought, but I know it's good. Just there are so many other things going on, it is a low priority (to take breaks)" (P7, female, clinical research admin, university/healthcare). For some participants, the goal to take regular breaks pertained to distal health outcomes, which, despite its prominence at the time of interview, might have to give way to more proximal goals that were mostly work-related in an office setting, e.g. "...because that's so long term, whereas you can see the short-term effects and take that on board more" (P13, female, clinical research admin, university/healthcare); "in my mode as I am NOW trying to be good, saying it is a good idea to break every 50 or 60 minutes, I am going to say if the display is there and it makes you break earlier then that is good. But if I am really into whatever I am doing, I would throw it out of the window or something" (P9, male, academic, university).

Lack of cognitive resources for regulating break behaviours at work . Apart from reflective motivation to adopt a healthier break pattern, a lack of cognitive resources for memory, attention and decision processes was another perceived barrier.

Firstly, participants reported the common experience of being "entrenched" or "engrossed" in work and forgetting to take breaks, e.g. "I lose track of time very easily, especially if I'm coding. I know afterwards when I look at the watch and I see that it's been 3 hours and I haven't moved" (P1, female, academic, university). Sometimes the concentration on work led to the neglect of physiological triggers for breaks, "if we have got 10 units of attention and 10 units focused on the screen on what we are writing, then we are not going to notice that your foot hurts" (P9). Delaying toilet and water breaks despite physiological needs was a common behaviour reported by participants, which will be discussed in relation to automatic motivation in the next subsection.

Secondly, people felt they lacked the memory capacity to remember how many breaks they had taken or how many episodes of prolonged sitting they had accumulated throughout the day. For instance, P6 (male, clinical research IT support, university/healthcare) said, "It (the diary) made me realise I'd taken a break that perhaps wouldn't normally even register in my head that I'd taken a break." This had implications for the belief about capability domain, as participants thought a physical record of breaks like the paper diary offered the reassurance that they were able to cut off potential prolonged sitting with simple microbreaks, "sometimes my breaks are so short that I didn't consider them as breaks. But I had to write them down. Then I thought, ah, that's a nice break. Even if it was 5 minutes, that's something" (P1). Visual feedback such as Fig. 1 could also facilitate self-reflection and potentially improve self-efficacy, "I think if the technology would be there, it would make me work much better to that pattern...especially if it would be something where I would look back on what I've done and then just review myself in actually you're improving or 
not improving on what I want to do" (P7, female, clinical research admin, university/healthcare).

Finally, decisions about whether to take a break or "power through" could become quite complex and dependent on many factors, such as progress in the current task, physical and mental fatigue, expectancy of outcome, next appointment arrangement etc. This was well illustrated by an incident reported by P18 (male, tech support/project management, university), "if I had a task I needed to complete for a meeting for 11:00 a.m., I'd look at that break reminder and go, 'right, am I going to get this stuff done for 11:00 a.m., if I have a cup of tea now?' I'd then either think, 'yes I am, I'll have a cup of tea because I know that the number of minutes after the break will be of a higher quality in terms of production and freshness than just pushing through' or I'd look and go 'you know what? It's going to be pretty damn close for me to finish, what I'll do is I'll keep going and have a cup of tea as a reward when I've done it.' It's a judgement call on an individual basis." However, given the complexity of the decision process and scarcity of cognitive resources at work, many break-related decisions were carried out "naturally" and "as a habit", as described in participants' diaries and interviews.

Habitual and emotional responses towards cues/prompts for breaks . 12 participants mentioned existing habits and routines that contributed to regular breaks. Some (P1, P7, P10, P13, P16) reported the habit of sipping water constantly while seated, so they were prompted to stand up every one to two hours to go to the toilet or/and refill vessels. The only smoker (P17, male, admin, university) in this study had the least accumulative prolonged sitting time, as he felt a strong impulse to take a cigarette break every hour. While smoking is certainly discouraged, these examples illustrated physiological needs could act as an efficient (i.e. require little attentional resources) and powerful (i.e. not easily controlled by intentionality) mechanism for instigating breaks and regulating overall break patterns.

On the other hand, automatic motivation could also work as higher-level mental processes [2] and unconscious self-regulation [1], which was manifested as habitual delay of breaks despite physiological needs for water and toilet breaks. Some described frequent moments when they put off breaks until reaching a natural "break point" in their task. In this case, some (P1, P13, P14, P16) had established a dependent relationship between natural "break points" in working tasks and the response to take a break; indeed, participants commonly described "micro-breaks" as rewards for completing a good amount of work. However, the risk with this contingency was that the scheduled work might take much longer than planned. e.g. "sometimes you plan it and then you don't plan it correctly and it takes a lot longer. It would've been good for a trigger at one hour to say this is when you should have stopped, you haven't, but you should have a break anyway" ( P14, female, clinical research admin, university/healthcare). This suggested the need for applying new "if-then" rules in breaking existing habits, and the usefulness of prompts/cues that were triggered after every certain minutes of sitting.

Modifying ingrained work patterns could also involve emotions. For instance, P6 reported that his prolonged sitting habit stemmed from 20 -year working history at a small private company, where "everything was urgent. If things broke down, they needed them repairing and you had to deal with it immediately", and this sense of urgency continued to influence 
his current work practice, "I think the biggest thing for me is to not feel so guilty if I'm running behind schedule". In contrast, P7 who took hourly micro-breaks most of the time, said, "because I know it is better for you, so I enjoy taking the breaks and don't feel guilty about it." This suggested affect could influence break behaviours in both directions and could act as a potential lever for change.

Organisational culture and interpersonal influences. While all participants in this study had freedom to take micro-breaks, organisational culture and the level of institutional control ranged from encouraging flexible, active and interactive work practices (e.g. "we are encouraged even, to be active and engage. You judge a person on what they do rather than how they look or where they are" (P18, male, tech support, university)), to no explicit expectation or surveillance (e.g. "I feel alright to take a break whenever, because they don't know what I'm doing as well" (P4, female, researcher, university/healthcare)), and monitoring and discouraging individuals leaving seats during office hours (e.g. "the manager will come and say where's so and so? They're not in a meeting according to my diary...Why aren't they sitting at their desks?" (P20, female, tech support, university)).

While it is challenging for a PT to directly change organisational climates, the study revealed the potential to make use of interpersonal influences on individual attitudes and behaviours. When participants were presented with the ranking based on healthiness of their own sitting patterns against those of others, they started comparing their own data with others', and making comments on both themselves and those at the bottom. The ranking might motivate them to "see how close to the top of this league you can get by having the appropriate number of breaks, never sitting for too long" (P9).

In addition to social comparison, social interactions directly affected break frequency. For example, some offices had the culture of inviting each other to make drinks together (P4, P5, P7, P12-15), whereas others had the practice of one person bringing drinks for the rest, who could remain seated and working for longer (e.g. P8, P11). Social support was another potential facilitator, e.g. "because we are in a caring environment and people do care about their colleague's health...so I think if you felt that somebody else had been sitting there for longer than is healthy then I think you could say something to them" (P19, female, clinical research admin, university/healthcare).

\subsection{Selection of intervention functions and BCTs}

In this section, we present our recommendation on intervention functions and BCTs that designers can consider when designing a PT to encourage regular micro-break behaviours. The selection was based on what the $\mathrm{BCW}$ suggests as potentially viable for addressing the 5 COM-B components and 11 TDF domains displayed in Table 2. A full result table can be download from http://dx.doi.org/10.17639/nott.72, which details how the identified barriers lead to recommendations that are exemplified with potential system features. As the BCTs are underpinned by behaviour change theories, PT designers need to refer to [13] for their full definitions when applying them.

Recommended intervention functions: Education, persuasion, training, enablement, environmental reconstructuring, modelling and incentivisation. 
Recommended BCTs: "Credible sources" "information about health consequences", "information about social and environmental consequences", "information about emotional consequences", "feedback on behaviour", "focus on past success", "verbal persuasion about capability", "information about other's approval”, "social comparison”, "behavioural practice", "habit formation", "adding objects to the environment", "prompts and cues", "conserving mental resources", "action planning", "goal setting", "self-monitoring of behaviour", "review behaviour goals", "monitoring of emotional consequences", "demonstration of the behaviour", "social reward", and "reward approximation".

\section{$5 \quad$ Conclusion and Recommendation}

In this study, we have presented a systematic diagnosis of office workers' perceived barriers to taking micro-work-breaks. As barriers identified fall into multiple domains, a singlefaceted intervention is insufficient to produce the desired behaviour change outcome. Therefore, for PT designers and practitioners whose aim is to implement a PT that is most likely to be effective, we suggest a multi-component intervention that incorporates as many of the recommended BCTs as possible, within the practical constraints imposed on them. That being said, we acknowledge the fact that our study is limited by the small sample size and potential self-selection bias, which means the identified determinants and recommended interventions may not be equally applicable to the whole population of office workers. Hence, we suggest PT designers consider our recommendations as potentially effective options informed by theories, but narrow the list down to design requirements most appropriate and feasible for different local contexts. A heuristic for doing this is to download the full result table, select the identified barriers supported with participants' quotes that seem to be most relevant to your local context, and thoroughly consider the corresponding BCTs and system features.

Methodologically speaking, we have demonstrated to the broader readership in the PT community a systematic approach, using the COM-B/TDF to diagnose a behaviour change problem and the $\mathrm{BCW}$ to translate the diagnosis into theory-informed design recommendations. We believe this approach does lend itself to a comprehensive coverage of factors in the exploratory phase of design research and for generation of new research directions and hypotheses; but the extent to which those factors and BCTs impact on the behaviour need to be tested with further experimental studies.

Acknowledgement . We would like to thank Kathryn Morgan and Rachael Travers for helping transcribe interviews, and Anna Roberts for reviewing the coding and intervention mapping. This research was supported by the Horizon Centre for Doctoral Training at the University of Nottingham (RCUK Grant No. EP/L015463/1) and by the RCUK's Horizon Digital Economy Research Institute (RCUK Grant No. EP/G065802/1) and Unilever UK Ltd. The study received ethics approval from School of Computer Science Ethics Committee, University of Nottingham. 


\section{Reference}

1. Aarts, H., Custers, R.: Unconscious Goal Pursuit: Nonconscious Goal Regulation and Motivation. In: The Oxford Handbook of Human Motivation. pp. 232-247 Oxford University Press, New York (2012).

2. Bargh, J. a, Ferguson, M.J.: Beyond behaviorism: on the automaticity of higher mental processes. Psychol. Bull. 126, 6, 925-45 (2000).

3. Cane, J. et al.: Validation of the theoretical domains framework for use in behaviour change and implementation research. Implement. Sci. 7, 1, 37 (2012).

4. Cirillo, F.: The Pomodoro Technique. FC Garage, Berlin (2013).

5. Consolvo, S. et al:: Theory-driven design strategies for technologies that support behavior change in everyday life. Proc. 27th Int. Conf. Hum. factors Comput. Syst. - CHI 09. 405-414 (2009).

6. Fogg, B.: A behavior model for persuasive design. In: The 4th International Conference on Persuasive Technology. p. 40 ACM Press, New York, New York, USA (2009).

7. Francis, J.J. et al.: Evidence-based selection of theories for designing behaviour change interventions: using methods based on theoretical construct domains to understand clinicians' blood transfusion behaviour. Br. J. Health Psychol. 14, Pt 4, 625-646 (2009).

8. French, S.D. et al.: Developing theory-informed behaviour change interventions to implement evidence into practice: a systematic approach using the Theoretical Domains Framework. Implement. Sci. 7, 1, 38 (2012).

9. Fritz, C. et al.: It's the little things that matter: An examination of knowledge workers' energy management. Acad. Manag. Perspect. 28-39 (2011).

10. Herrmanny, K. et al.: Supporting Users in Setting Effective Goals in Activity Tracking. In: The 11th International Conference on Persuasive Technology. pp. 15-26 Springer-Verlag New York, Inc. (2016).

11. Jett, Q.R., George, J.M.: Work interrupted: A closer look at the role of interruptions in organizational life, (2003).

12. Mark, G. et al.: No Task Left Behind ? Examining the Nature of Fragmented Work. CHI 2005 Proc. SIGCHI Conf. Hum. Factors Comput. Syst. 321-330 (2005).

13. Michie, S. et al.: The Behaviour Change Wheel: A Guide to Designing Interventions. Silverback Publishing (2014).

14. Owen, N. et al.: Too much sitting: a novel and important predictor of chronic disease risk? Br. J. Sports Med. 43, 2, 81-3 (2009).

15. Pate, R.R. et al.: The evolving definition of "sedentary". Exerc. Sport Sci. Rev. 36, 4, 173-178 (2008).

16. Prochaska, J.O., Velicer, W.F.: The transtheoretical model of health behavior change. Am. J. Heal. Promot. AJHP. 12, 1, 38-48 (1997).

17. Ryan, C.G. et al.: Sitting patterns at work: objective measurement of adherence to current recommendations. Ergonomics. 54, 6, 531-538 (2011).

18. Srivastava, A., Thomson, S.B.: Framework Analysis: A qualitative methodology for applied policy research. J. Adm. Gov. 4, 2, 72-79 (2009).

19. Tremblay, M.S. et al.: Physiological and health implications of a sedentary lifestyle. Appl. Physiol. Nutr. Metab. 35, 6, 725-740 (2010).

20. West, R.: Time for a change : putting theTranstheoretical ( Stages of Change ) Model to rest. Addiction. 1036-1040 (2005).

21. Züger, M., Fritz, T.: Interruptibility of Software Developers and its Prediction Using PsychoPhysiological Sensors. Proc. 33rd Annu. ACM Conf. Hum. Factors Comput. Syst. - CHI '15. 2981-2990 (2015). 